Groups Geom. Dyn. 5 (2011), 367-391

DOI $10.4171 / \mathrm{GGD} / 132$
Groups, Geometry, and Dynamics

(C) European Mathematical Society

\title{
On the Arthur-Selberg trace formula for GL(2)
}

\author{
Tobias Finis and Erez Lapid*
}

To the memory of Fritz, Grunewald

\begin{abstract}
We extend the terms on the geometric side of the trace formula for GL(2) over $\mathbb{Q}$ continuously to a natural Fréchet algebra of non-compactly supported test functions. For the spectral side the analogous result had been obtained previously (in much greater generality) in collaboration with W. Müller.
\end{abstract}

Mathematics Subject Classification (2010). 11F72, 11F41.

Keywords. Trace formula, automorphic forms.

\section{Introduction}

The trace formula is one of the most important tools in the theory of automorphic forms. It was introduced by Selberg in his celebrated paper [Sel56] to study the spectra of locally symmetric spaces $\Gamma \backslash G / K$, where $\Gamma$ is a lattice in a reductive Lie group $G$ and $K$ a maximal compact subgroup of $G$. More generally, one can consider the right regular representation of $G$ on the space $L^{2}(\Gamma \backslash G)$. For functions $f \in L^{1}(G)$ this representation induces the integral operators

$$
R(f) \varphi(x)=\int_{G} f(g) \varphi(x g) d g .
$$

The derivation of the trace formula identity is not difficult in the case of uniform lattices $\Gamma$, i.e. when the quotient space $\Gamma \backslash G$ is compact. (We remark that only in this case Selberg was able to treat general groups $G$.) The operator $R(f)$ is then of trace class for $f \in C_{c}^{\infty}(G)$ and $L^{2}(\Gamma \backslash G)$ decomposes discretely as $\bigoplus_{\pi \in \hat{G}} m(\pi) \pi$. The trace of $R(f)$ can be evaluated as

$$
\sum_{[\gamma]} \operatorname{vol}\left(\Gamma_{\gamma} \backslash G_{\gamma}\right) \int_{G_{\gamma} \backslash G} f\left(g^{-1} \gamma g\right) d g=\sum_{\pi \in \hat{G}} m(\pi) \operatorname{tr} \pi(f)
$$

*Authors partially sponsored by grant \# 964-107.6/2007 from the German-Israeli Foundation for Scientific Research and Development. This paper forms a part of the first named author's 2009 Habilitation at Heinrich-Heine-Universität Düsseldorf. 
where $\gamma$ ranges over the conjugacy classes of $\Gamma$ and $\Gamma_{\gamma}$ is the centralizer of $\gamma$ in $\Gamma$. This is Selberg's trace formula in the case of compact quotient. It can be extended from $C_{c}^{\infty}(G)$ to the space of smooth functions on $G$ whose derivatives are all in $L^{1}(G)$.

Selberg transformed the trace formula for bi- $K$-invariant test functions $f$ into an identity between distributions on the space of spherical spectral parameters, which is reminiscent of the Poisson summation formula in that it involves (weighted) delta distributions and their Fourier transforms (in addition to more general distributions, for example the contribution from the identity element). The analytic applications of the formula include Weyl's law and its multi-variable generalizations by Duistermaat-Kolk-Varadarajan ([DKV79]), which describe the asymptotic behavior of the spectrum of $\Gamma \backslash G / K$, and the results on limit multiplicities obtained by DeGeorge-Wallach ([DW78], [DW79]) and Delorme ([Del86]) among others. It is also possible to incorporate the action of double classes $\Gamma \gamma \Gamma$, where $\gamma$ is an element of the commensurator of $\Gamma$ in $G$ (Hecke operators).

Selberg also extended the trace formula to the case of non-cocompact lattices $\Gamma$ in the group $\operatorname{SL}(2, \mathbb{R})$. In this case the operators $R(f)$ are not of trace class and the spectrum is not discrete. Nevertheless, it is possible to derive a trace formula identity which includes modified terms for unipotent conjugacy classes in $\Gamma$ and a contribution from the continuous spectrum on the other side. Selberg used his formula to deduce Weyl's law for congruence subgroups of $\operatorname{SL}(2, \mathbb{Z})$.

A necessary prerequisite for the trace formula is an understanding of the spectral decomposition of the space $L^{2}(\Gamma \backslash G)$. Selberg initiated the theory and worked out the case of lattices in $\operatorname{SL}(2, \mathbb{R})$. The higher rank case was successfully treated in the groundbreaking work of Langlands ([Lan76]). Building on this, Arthur considered in his lifelong work the trace formula in the context of adelic quotients $G(F) \backslash G(\mathbb{A})$ of general reductive groups $G$ over number fields $F$. (Compared to the above formulation, this amounts to the consideration of all corresponding congruence arithmetic lattices in $G(F) \subseteq G(F \otimes \mathbb{R})$ and all possible Hecke operators at once.) Arthur's trace formula is an identity

$$
J_{\text {geom }}(f)=J_{\text {spec }}(f)
$$

between a sum of geometric distributions and a sum of spectral distributions on the group $G(\mathbb{A})^{1}$. The test functions $f$ are compactly supported smooth functions on $G(\mathbb{A})^{1}$. The main task undertaken by Arthur after establishing this identity was to explicate these distributions (albeit not completely). See [Art05] for more details and a broader scope of the trace formula and for a survey of its deep applications in the context of the Langlands program.

It is natural to ask whether there is a bigger class of test functions to which the trace formula applies. More precisely, can we find a "natural" Fréchet algebra of functions on $G(\mathbb{A})^{1}$ (or perhaps a direct limit of such algebras) containing $C_{c}^{\infty}\left(G(\mathbb{A})^{1}\right)$ as a dense subalgebra, for which the sum-integrals appearing on both sides of the 
trace formula are absolutely convergent and define (if taken with absolute values) continuous seminorms?

A case to bear in mind is the Poisson summation formula

$$
\sum_{n \in \mathbb{Z}} f(n)=\sum_{n \in \mathbb{Z}} \hat{f}(n)
$$

which is valid for functions $f$ on $\mathbb{R}$ whose derivative is absolutely continuous and for which $f, f^{\prime}, f^{\prime \prime} \in L^{1}(\mathbb{R})$. The space of these functions forms a Banach algebra (Sobolev space) with respect to the norm $\|f\|_{1}+\left\|f^{\prime}\right\|_{1}+\left\|f^{\prime \prime}\right\|_{1}$, where $\|\cdot\|_{1}$ denotes the $L^{1}$-norm. Moreover,

$$
\begin{gathered}
\sum_{n \in \mathbb{Z}}|f(n)| \leq\|f\|_{1}+\left\|f^{\prime}\right\|_{1}, \\
\sum_{n \in \mathbb{Z}}|\hat{f}(n)| \leq \sum_{n \in \mathbb{Z}} \frac{1}{(2 \pi n)^{2}+1}\left(\|f\|_{1}+\left\|f^{\prime \prime}\right\|_{1}\right)=\left(\frac{1}{2}+\frac{1}{e-1}\right)\left(\|f\|_{1}+\left\|f^{\prime \prime}\right\|_{1}\right) .
\end{gathered}
$$

Analogously, natural Fréchet algebras of test functions for Arthur's trace formula are obtained by fixing a compact open subgroup $K$ of $G\left(\mathbb{A}_{\text {fin }}\right)$ and considering bi$K$-invariant functions on $G(\mathbb{A})^{1}$. The double coset space $K \backslash G(\mathbb{A})^{1} / K$ is a disjoint union of countably many copies of $G(\mathbb{R})^{1}=G(\mathbb{R}) \cap G(\mathbb{A})^{1}$, and in particular a differentiable manifold. Let $\mathfrak{g}_{\mathbb{C}}^{1}$ denote the complexified Lie algebra of $G(\mathbb{R})^{1}$ and $\mathcal{U}\left(\mathfrak{g}_{\mathbb{C}}^{1}\right)$ its universal enveloping algebra with the usual grading. This algebra acts from the left and from the right on the space of smooth functions on $K \backslash G(\mathbb{A})^{1} / K$. We consider the Fréchet algebra $\mathcal{C}\left(G(\mathbb{A})^{1} ; K\right)$ of smooth functions $f$ on $K \backslash G(\mathbb{A})^{1} / K$ for which the norms

$$
\|f\|_{n}:=\sum_{i, j \geq 0, i+j \leq n} \sum_{X \in \mathcal{B}_{i}, Y \in \mathcal{B}_{j}}\|X * f * Y\|_{1}, \quad n=0,1, \ldots,
$$

are finite, where $\mathscr{B}_{k}$ denotes a basis of $U\left(\mathfrak{g}_{\mathbb{C}}^{1}\right)_{k}$. The subalgebra $C_{c}^{\infty}\left(G(\mathbb{A})^{1} ; K\right)$ of compactly supported smooth bi- $K$-invariant functions on $G(\mathbb{A})^{1}$ is dense in $\varphi\left(G(\mathbb{A})^{1} ; K\right)$.

Non-compactly supported test functions appeared already in Selberg's work. Important examples in spectral theory are the heat kernels. In the adelic framework, this corresponds to test functions with non-compact support at the infinite places. Another motivation emerged from a relatively recent idea of Langlands to use the trace formula in order to sieve the representations having a pole at $s=1$ for a given $L$-function ([Lan04], [Lan07]). This requires the consideration of test functions whose support is non-compact at almost all places. (See (11), (12) below for a simple example.) A closely related setup of limiting forms of the trace formula shows up in the thesis of Akshay Venkatesh ([Ven04]).

In the paper [FLM], W. Müller and the present authors refined the spectral side of Arthur's trace formula and at the same time extended it to test functions in 
$\mathcal{C}\left(G(\mathbb{A})^{1} ; K\right)$. We quickly sketch the main result, referring to [loc. cit.] for details. ${ }^{1}$ The basic objects appearing on the spectral side are the representations of $G(\mathbb{A})$ parabolically induced from the discrete spectra of the Levi subgroups $M$ of $G$. For a parabolic subgroup $P$ of $G$ with Levi decomposition $P=M U$ let $a_{M}^{*}$ denote the real vector space spanned by the $F$-rational characters of $G / Z(G), a_{M}$ the dual space, and $H_{P}: G(\mathbb{A}) \rightarrow a_{M}$ the map induced by the Iwasawa decomposition $G(\mathbb{A})=M(\mathbb{A}) U(\mathbb{A}) \boldsymbol{K}$ with respect to a suitable maximal compact subgroup $\boldsymbol{K}$ of $G(\mathbb{A})$. Let

$$
\left(\overline{\mathcal{A}}^{2}(P), \rho(P, \lambda)\right), \quad \lambda \in \mathrm{a}_{M}^{*} \otimes \mathbb{C},
$$

be the representation of $G(\mathbb{A})$ induced from the $P(\mathbb{A})$-representation

$$
L_{\mathrm{disc}}^{2}\left(A_{M} M(\mathbb{Q}) \backslash M(\mathbb{A})\right) \otimes e^{\left\langle\lambda, H_{P}(\cdot)\right\rangle} .
$$

The representation $\rho(P, \lambda)$ is unitary for $\lambda \in \mathrm{ia}_{M}^{*}$. In Arthur's formulation the spectral side involves the representations $\rho(P, \lambda)$ for $\lambda \in \mathrm{i} a_{M}^{*}$, but it is written as an infinite sum over contributions associated to so-called cuspidal data because of a subtle convergence problem. In [loc. cit.], this problem was resolved and the spectral side could be rewritten in the form

$$
\begin{aligned}
J_{\text {spec }}(f)= & \sum_{[M]} \frac{1}{\left[N_{G}(M): M\right]} \sum_{s \in N_{G}(M) / M}|\operatorname{det}(s-1)|_{\mathfrak{a}_{M} / \mathfrak{a}_{M}^{s}}^{-1} \\
& \int_{i\left(\mathfrak{a}_{M}^{*}\right)^{s}} \operatorname{tr}\left(\mathcal{M}_{M_{s}}(P, \lambda) M_{P \mid P}(s, 0) \rho(P, \lambda, f)\right) d \lambda
\end{aligned}
$$

for $f \in \mathscr{C}\left(G(\mathbb{A})^{1} ; K\right)$, where the sum is over conjugacy classes of Levi subgroups $M$ of $G$ and the Levi subgroup $M_{s}$ is defined by $a_{M_{s}}=\mathfrak{a}_{M}^{s}$, the space of vectors in $a_{M}$ fixed by $s$. The operators $M_{P \mid P}(s, 0)$ are intertwining operators and the operators $\mathcal{M}_{M_{S}}$ multi-dimensional derivatives of intertwining operators acting on the space $\overline{\mathcal{A}}^{2}(P)$. It is also possible to express $\mathcal{M}_{M_{s}}$ in terms of logarithmic derivatives of co-rank one intertwining operators. Furthermore, if one replaces the trace by the trace norm, the integral-sum defines a continuous seminorm on $\ell\left(G(\mathbb{A})^{1} ; K\right)$.

It is natural to expect an analog of this result for the geometric side, resulting in a trace formula identity which is valid for functions in $\mathcal{C}\left(G(\mathbb{A})^{1} ; K\right)$. One encounters two principal difficulties with Arthur's fine geometric expansion ([Art85], [Art86]), in which $J_{\text {geom }}(f)$ is expressed as a sum over conjugacy classes of Levi subgroups $M$ and classes of elements $\gamma \in M(F)$ (for a certain equivalence relation weaker than conjugacy and depending on a finite set of places $S$ ) of constant multiples of weighted orbital integrals $J_{M}(\gamma, f)$. First, it applies only to functions of the form $f_{S} \otimes \mathbf{1}^{S}$ where $S$ is a finite set of places (containing the Archimedean ones) and $\mathbf{1}^{S}$ is the characteristic function of the maximal compact subgroup $\boldsymbol{K}^{S}$ outside $S$ (rather

\footnotetext{
${ }^{1}$ A preliminary announcement is contained in the note [FLM09]. The Fréchet algebras considered in these two papers are slightly different from the Fréchet algebras $\mathcal{C}\left(G(\mathbb{A})^{1} ; K\right)$.
} 
than an arbitrary bi- $\boldsymbol{K}^{S}$-invariant function outside $S$ ). The second and more serious difficulty is that while the local distributions $J_{M}(\gamma, \cdot)$ appearing in the expansion are explicit and fairly well-understood, their coefficients are left unspecified, and depend on $S$ in a complicated way. For the problem at hand it would be imperative to bound them in a uniform way.

The purpose of this paper is to resolve the problem for the group $G=$ GL(2) over $\mathbb{Q}$, where a completely explicit form of the trace formula is available (see [GJ79], [Gel96], [KL06] for detailed accounts). The restriction to $\mathbb{Q}$ is merely for the sake of exposition. We give an extension of the trace formula to $\mathcal{C}\left(G(\mathbb{A})^{1} ; K\right)$ and prove the continuity of the associated seminorms. The precise statement is given by Theorem 1 below. The terms in the extended trace formula associated to the non-elliptic conjugacy classes are modified compared to the usual trace formula for $C_{c}^{\infty}\left(G(\mathbb{A})^{1} ; K\right)$. This is crucial because the contribution of the unipotent classes is originally defined only for compactly supported functions. We also write the regular hyperbolic contribution in a structurally analogous way. The non-elliptic terms in the modified trace formula depend on a finite set of places $S$ with $K \supseteq \boldsymbol{K}^{S}$. The modified distributions appearing in these terms are an interesting feature of our version of the trace formula. Note that even if we are interested only in compactly supported test functions, our formula is by construction independent of the support of the test function.

We hope that the case of GL(2) will serve as a blueprint for the general case. In fact, since the first version of this paper was completed, the authors have succeeded in establishing the continuity of the semisimple part of the trace formula for general reductive groups $G$ ([FL]). ${ }^{2}$ We would also like to mention related work by Hoffmann on the coarse geometric expansion ([Hof08]).

We thank the referee for a careful reading of the manuscript which led to the correction of several inaccuracies.

\section{The main result}

Notation. Let $\mathbb{A}=\mathbb{R} \times \mathbb{A}_{\text {fin }}$ be the ring of adeles, $\mathbb{I}$ (resp. $\mathbb{I}^{1}$ ) the group of ideles (resp. ideles of norm 1), and $\mathcal{O}=\prod_{p<\infty} \mathbb{Z}_{p}$. Let $G$ be the group GL(2), $Z$ its center, $B$ the standard Borel subgroup, $T$ the diagonal torus and $U$ the unipotent radical of $B$. Let $\boldsymbol{K}=\prod_{p \leq \infty} \boldsymbol{K}_{p}$ be the standard maximal compact subgroup of $G(\mathbb{A})$.

Set $G(\mathbb{A})^{1}=\{g \in G(\mathbb{A}):|\operatorname{det} g|=1\}, Z(\mathbb{A})^{1}=Z(\mathbb{A}) \cap G(\mathbb{A})^{1}$ and $T(\mathbb{A})^{1}=$ $\left\{\left(\begin{array}{cc}t_{1} & 0 \\ 0 & t_{2}\end{array}\right): t_{1}, t_{2} \in \mathbb{I}^{1}\right\}$. We have

$$
\begin{gathered}
G(\mathbb{A}) \simeq G(\mathbb{A})^{1} \times A_{G}, \quad T(\mathbb{A})=\left(T(\mathbb{A}) \cap G(\mathbb{A})^{1}\right) \times A_{G}, \\
T(\mathbb{A})=T(\mathbb{A})^{1} \times A_{0},
\end{gathered}
$$

where $A_{G}=\left\{\left(\begin{array}{cc}a & 0 \\ 0 & a\end{array}\right): a \in \mathbb{R}_{>0}\right\}$ and $A_{0}=\left\{\left(\begin{array}{cc}a_{1} & 0 \\ 0 & a_{2}\end{array}\right): a_{1}, a_{2} \in \mathbb{R}_{>0}\right\}$.

\footnotetext{
${ }^{2}$ The Fréchet algebras considered in [FL] are slightly different from the Fréchet algebras $e\left(G(\mathbb{A})^{1} ; K\right)$.
} 
We fix Haar measures according to the following table:

\begin{tabular}{|c|c|}
\hline group & normalization \\
\hline discrete & counting measure \\
\hline $\mathbb{R}$ & Lebesgue measure \\
\hline$i \mathbb{R}$ & through $x \mapsto \mathrm{i} x$ \\
\hline $\mathbb{Q}_{p}, p<\infty$ & $\operatorname{vol}\left(\mathbb{Z}_{p}\right)=1$ \\
\hline $\mathbb{A}$ & product measure \\
\hline $\mathbb{R}^{*}, \mathbb{R}_{>0}$ & $d^{*} x=\frac{d x}{|x|}$ \\
\hline $\mathbb{Q}_{p}^{*}, p<\infty$ & $\operatorname{vol}\left(\mathbb{Z}_{p}^{*}\right)=1$ \\
\hline $\mathbb{I}$ & product measure \\
\hline $\mathbb{I}^{1}$ & compatible with $\mathbb{I}=\mathbb{R}_{>0} \times \mathbb{I}^{1}$ \\
\hline$Z(\mathbb{A})^{1}, A_{G}$ & through $t \mapsto\left(\begin{array}{cc}t & 0 \\
0 & t\end{array}\right)$ \\
\hline$U\left(\mathbb{Q}_{p}\right), U(\mathbb{A})$ & through $x \mapsto\left(\begin{array}{ll}1 & x \\
0 & 1\end{array}\right)$ \\
\hline$T\left(\mathbb{Q}_{p}\right), T(\mathbb{A}), T(\mathbb{A})^{1}, A_{0}$ & through $t_{1}, t_{2} \mapsto\left(\begin{array}{cc}t_{1} & 0 \\
0 & t_{2}\end{array}\right)$ \\
\hline $\boldsymbol{K}_{p}, p \leq \infty, \boldsymbol{K}$ & $\mathrm{vol}=1$ \\
\hline$G\left(\mathbb{Q}_{p}\right), p \leq \infty, G(\mathbb{A})$ & compatible with Iwasawa decomposition \\
\hline$G(\mathbb{A})^{1}, T(\mathbb{A}) \cap G(\mathbb{A})^{1}$ & compatible with (1) \\
\hline
\end{tabular}

Let

$$
\zeta^{*}(s)=\int_{\mathbb{I}} e^{-\pi x_{\infty}^{2}} \chi_{\mathbb{I} \cap \mathcal{O}}\left(x_{\mathrm{fin}}\right)|x|^{s} d^{*} x=\pi^{-s / 2} \Gamma(s / 2) \prod_{p<\infty}\left(1-p^{-s}\right)^{-1} .
$$

Recall that $\operatorname{vol}(\mathbb{Q} \backslash \mathbb{A})=1$ and $\operatorname{vol}\left(\mathbb{Q}^{*} \backslash \mathbb{I}^{1}\right)=\operatorname{res}_{s=1} \zeta^{*}(s)(=1)$.

The modulus function on $B(\mathbb{A})$ is given by $\delta_{B(\mathbb{A})}\left(\left(\begin{array}{cc}t_{1} & 0 \\ 0 & t_{2}\end{array}\right)\right)=\left|\frac{t_{1}}{t_{2}}\right|$. If $g \in G(\mathbb{A})$ has Iwasawa decomposition $g=t u k, t=\left(\begin{array}{cc}t_{1} & 0 \\ 0 & t_{2}\end{array}\right) \in T(\mathbb{A}), u \in U(\mathbb{A}), k \in \boldsymbol{K}$, we write $\mathrm{t}(g)=\left|\frac{t_{1}}{t_{2}}\right| \in \mathbb{R}_{>0}$. Similarly $\overline{\mathrm{t}}$ is defined with respect to the opposite Borel subgroup $\bar{B}=T \bar{U}$. Recall that

$$
\mathrm{t}(g) \leq \overline{\mathrm{t}}(g)
$$

for all $g \in G(\mathbb{A})$.

We denote by $R_{\text {disc }}(f)$ the action of $L^{1}\left(G(\mathbb{A})^{1}\right)$ on the discrete part of $L^{2}\left(G(\mathbb{Q}) \backslash G(\mathbb{A})^{1}\right)$. Consider the space

$$
\mathfrak{I}=\operatorname{Ind}_{B(\mathbb{A})}^{G(\mathbb{A})} L^{2}\left(A_{0} T(\mathbb{Q}) \backslash T(\mathbb{A})\right)
$$

which is the completion of the space of smooth functions $\varphi: G(\mathbb{A}) \rightarrow \mathbb{C}$ such that $\varphi(u t a g)=\delta_{B(\mathbb{A})}^{\frac{1}{2}}(a) \varphi(g)$ for all $u \in U(\mathbb{A}), t \in T(\mathbb{Q}), a \in A_{0}$ and $g \in G(\mathbb{A})$ and

$$
\|\varphi\|^{2}=\int_{A_{0} U(\mathbb{A}) T(\mathbb{Q}) \backslash G(\mathbb{A})}|\varphi(g)|^{2} d g=\int_{K} \int_{T(\mathbb{Q}) \backslash T(\mathbb{A})^{1}}|\varphi(t k)|^{2} d t d k<\infty .
$$


For any $s \in \mathbb{C}$ the group $G(\mathbb{A})^{1}$ acts on this space via

$$
I(g, s) \varphi(x)=\varphi(x g) \mathrm{t}(x g)^{s} \mathrm{t}(x)^{-s} .
$$

We denote by $I(f, s)$ the corresponding action of $L^{1}\left(G(\mathbb{A})^{1}\right)$. The theory of Eisenstein series gives rise to intertwining operators $M(s)$ which after meromorphic continuation define unitary operators on $\mathfrak{\Im}$ for $s \in \mathrm{i} \mathbb{R}$.

From now on, we fix an open subgroup $K$ of $\boldsymbol{K}_{\text {fin }}=\prod_{p<\infty} \boldsymbol{K}_{p}$. Let $S$ be a finite set of primes, including $\infty$, such that $K \supseteq \boldsymbol{K}_{p}$ if $p \notin S$. Define

$$
\lambda_{t, S}=-\sum_{p \notin S:\left|t_{1}\right|_{p}=\left|t_{2}\right|_{p}} \frac{1-\left|1-\frac{t_{2}}{t_{1}}\right|_{p}}{p-1} \log p
$$

for $t \in T(\mathbb{Q}) \backslash Z(\mathbb{Q})$ and

$$
\lambda_{z, S}=\lim _{X \rightarrow \infty}\left[\frac{\sum_{1 \leq n \leq X:(n, S)=1} \frac{1}{n}}{\operatorname{res}_{s=1} \zeta^{S}(S)}-\log X\right]
$$

for $z \in Z(\mathbb{Q})$, where we write $(n, S)=1$ to mean that $(n, p)=1$ for all $p \in S$, and

$$
\zeta^{S}(s)=\prod_{p \notin S}\left(1-p^{-s}\right)^{-1}=\sum_{n \geq 1:(n, S)=1} n^{-s}
$$

as usual. Note that if $p \notin S$ then for all $t \in T(\mathbb{Q})$ (including $Z(\mathbb{Q})$ ) we have

$$
\lambda_{t, S \cup\{p\}}=\lambda_{t, S}+ \begin{cases}\frac{1-\left|1-\frac{t_{2}}{t_{1}}\right|_{p}}{p-1} \log p, & \left|t_{1}\right|_{p}=\left|t_{2}\right|_{p}, \\ 0 & \text { otherwise. }\end{cases}
$$

We also remark that for $z \in Z(\mathbb{Q})$

$$
\lambda_{z, S}=\frac{\left[(s-1) \zeta^{S}(s)\right]_{s=1}^{\prime}}{\operatorname{res}_{s=1} \zeta^{S}(s)} .
$$

In fact, the relation (3) reduces this equality to the case $S=\{\infty\}$ which is a standard identity. (Both sides are equal to Euler's constant $\gamma$ in this case.)

Also define, for $t=\left(\begin{array}{cc}t_{1} & 0 \\ 0 & t_{2}\end{array}\right) \in T(\mathbb{A})$ and $u \in U(\mathbb{A})$,

$$
\omega(t, u)=\sum_{p \leq \infty} \omega_{p}(t, u)
$$

where for $p \in S$

$$
\omega_{p}\left(t,\left(\begin{array}{ll}
1 & x \\
0 & 1
\end{array}\right)\right)=\omega_{p}^{\mathrm{std}}\left(t,\left(\begin{array}{ll}
1 & x \\
0 & 1
\end{array}\right)\right):= \begin{cases}\log \max \left(|x|_{p},\left|1-\frac{t_{2}}{t_{1}}\right|_{p}\right), & p<\infty, \\
\log \sqrt{\left(1-\frac{t_{2}}{t_{1}}\right)^{2}+x^{2}}, & p=\infty,\end{cases}
$$


while for $p \notin S$

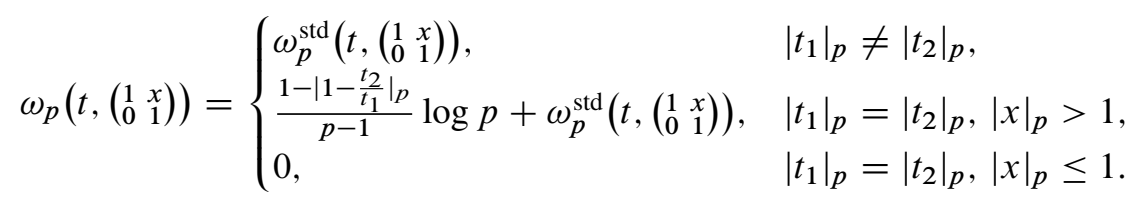

Note that for almost all $u \in U(\mathbb{A})$ (namely, when $x_{p} \neq 0$ for all $\left.p \in S\right) \omega(\cdot, u)$ is a continuous function on $T(\mathbb{A})$.

Finally, for any $\gamma \in G(\mathbb{Q})$, let $G_{\gamma}$ be the centralizer of $\gamma$, and let $G_{\gamma}(\mathbb{A})^{1}=$ $G_{\gamma}(\mathbb{A}) \cap G(\mathbb{A})^{1}$.

\section{The trace formula identity}

Theorem 1. For any $f \in \mathcal{C}\left(G(\mathbb{A})^{1} ; K\right)$

$$
\begin{gathered}
\sum_{\gamma \text { elliptic conjugacy class }} \operatorname{vol}\left(G_{\gamma}(\mathbb{Q}) \backslash G_{\gamma}(\mathbb{A})^{1}\right) \int_{G_{\gamma}(\mathbb{A}) \backslash G(\mathbb{A})} f\left(x^{-1} \gamma x\right) d x \\
+\operatorname{vol}\left(T(\mathbb{Q}) \backslash T(\mathbb{A})^{1}\right) \sum_{t \in T(\mathbb{Q})} \int_{K} \int_{U(\mathbb{A})} f\left(k^{-1} t u k\right) \omega(t, u) d u d k \\
+\operatorname{vol}\left(T(\mathbb{Q}) \backslash T(\mathbb{A})^{1}\right) \sum_{t \in T(\mathbb{Q})} \lambda_{t, S} \int_{\boldsymbol{K}} \int_{U(\mathbb{A})} f\left(k^{-1} t u k\right) d u d k \\
=\operatorname{tr} R_{\mathrm{disc}}(f)-\frac{1}{4 \pi} \int_{\mathrm{i} \mathbb{R}} \operatorname{tr}\left(M^{-1}(s) M^{\prime}(s) I(f, s)\right) d s+\frac{1}{4} \operatorname{tr}(M(0) I(f, 0)) .
\end{gathered}
$$

Moreover,

$$
\begin{gathered}
\sum_{\gamma \text { elliptic conjugacy class }} \operatorname{vol}\left(G_{\gamma}(\mathbb{Q}) \backslash G_{\gamma}(\mathbb{A})^{1}\right) \int_{G_{\gamma}(\mathbb{A}) \backslash G(\mathbb{A})}\left|f\left(x^{-1} \gamma x\right)\right| d x, \\
\sum_{t \in T(\mathbb{Q})} \int_{\boldsymbol{K}} \int_{U(\mathbb{A})}\left|f\left(k^{-1} t u k\right)\right| \sum_{p}\left|\omega_{p}(t, u)\right| d u d k, \\
\sum_{t \in T(\mathbb{Q})}\left|\lambda_{t, S}\right| \int_{\boldsymbol{K}} \int_{U(\mathbb{A})}\left|f\left(k^{-1} t u k\right)\right| d u d k,
\end{gathered}
$$

and

$$
\begin{gathered}
\left\|R_{\mathrm{disc}}(f)\right\|_{1}+\int_{\mathrm{i} \mathbb{R}}\left\|M^{-1}(s) M^{\prime}(s) I(f, s)\right\|_{1} d s+\|M(0) I(f, 0)\|_{1} \\
=\left\|R_{\mathrm{disc}}(f)\right\|_{1}+\int_{\mathrm{i} \mathbb{R}}\left\|M^{\prime}(s) I(f, s)\right\|_{1} d s+\|I(f, 0)\|_{1}
\end{gathered}
$$

are continuous seminorms on $\mathcal{C}\left(G(\mathbb{A})^{1} ; K\right)$, where in (8) the subscript 1 denotes trace norm. 
Implicit in the statement is that the operator $M^{-1}(s) M^{\prime}(s) I(f, s)$, originally defined on a dense subspace of $\mathfrak{I}$, extends to a bounded, and in fact trace-class operator. We also remark that

$$
\operatorname{tr}(M(0) I(f, 0))=-\left.\operatorname{tr} I(f, 0)\right|_{\mathfrak{\Im}}
$$

where

$$
\tilde{\Im}=\left\{\varphi \in \mathfrak{I}: \varphi\left(\left(\begin{array}{cc}
t & 0 \\
0 & t^{-1}
\end{array}\right) g\right)=\varphi(g) \text { for all } t \in \mathbb{I}^{1}\right\} .
$$

We will momentarily check that the identity of Theorem 1 reduces to the usual trace formula for GL(2) for $f \in C_{c}^{\infty}\left(G(\mathbb{A})^{1} ; K\right)$. This will reduce Theorem 1 to its continuity part which will be proved in $\S 4$. We point out however, that unlike in the usual trace formula for $f \in C_{c}^{\infty}\left(G(\mathbb{A})^{1}\right)$, the coefficients $\lambda_{t, S}$ and weights $\omega(t, x)$ appearing in the geometric side depend on the choice of $S$, and therefore, on $K$. This "feature" seems essential for our approach. Of course, for Theorem 1 to hold, the geometric side as a whole should not depend on the choice of $S$. To check that this is indeed the case, one only has to verify the purely local identity

$$
\int_{U\left(\mathbb{Q}_{p}\right)} f_{p}(t u)\left(\omega_{p}(t, u)+\lambda_{t, S}\right) d u=\int_{U\left(\mathbb{Q}_{p}\right)} f_{p}(t u)\left(\omega_{p}^{\mathrm{std}}(t, u)+\lambda_{t, S \cup\{p\}}\right) d u
$$

for $p \notin S$ and any bi- $\boldsymbol{K}_{p}$-invariant function $f_{p} \in L^{1}\left(G\left(\mathbb{Q}_{p}\right)\right)$. See Remark 1 in $\S 3$ for the necessary computation.

Assume now that $f \in C_{c}^{\infty}\left(G(\mathbb{A})^{1} ; K\right)$. Traditionally, the non-elliptic contribution is written in a different form than the above (cf. [GJ79], [Gel96], [KL06]; in the case at hand we sum over all characters of $\left.Z(\mathbb{Q}) \backslash Z(\mathbb{A})^{1}\right)$. Namely, the regular hyperbolic contribution is written as the sum over $t \in T(\mathbb{Q}) \backslash Z(\mathbb{Q})$ of

$$
\frac{1}{2} \operatorname{vol}\left(T(\mathbb{Q}) \backslash T(\mathbb{A})^{1}\right) \int_{T(\mathbb{A}) \backslash G(\mathbb{A})} f\left(x^{-1} t x\right)(\log \overline{\mathrm{t}}(x)-\log \mathrm{t}(x)) d x
$$

and the unipotent (modulo center) contribution as the derivative at $s=1$ of

$$
(s-1) \int_{\mathbb{I}} \Phi(x)|x|^{s} d^{*} x,
$$

where

$$
\Phi(x)=\operatorname{vol}\left(Z(\mathbb{Q}) \backslash Z(\mathbb{A})^{1}\right) \sum_{z \in Z(\mathbb{Q})} \int_{\boldsymbol{K}} f\left(k^{-1} z\left(\begin{array}{ll}
1 & x \\
0 & 1
\end{array}\right) k\right) d k .
$$

In order to see that this agrees with Theorem 1 , we use the Iwasawa decomposition to write the regular hyperbolic contribution as the sum over $t=\left(\begin{array}{cc}t_{1} & 0 \\ 0 & t_{2}\end{array}\right) \in T(\mathbb{Q}) \backslash Z(\mathbb{Q})$ of

$$
\frac{1}{2} \operatorname{vol}\left(T(\mathbb{Q}) \backslash T(\mathbb{A})^{1}\right) \int_{\boldsymbol{K}} \int_{U(\mathbb{A})} f\left(k^{-1} u^{-1} t u k\right) \log \overline{\mathrm{t}}(u) d u d k
$$


which by a change of variable is equal to

$$
\begin{aligned}
\frac{1}{2} \operatorname{vol}\left(T(\mathbb{Q}) \backslash T(\mathbb{A})^{1}\right) & \int_{\boldsymbol{K}} \int_{\mathbb{A}} f\left(k^{-1} t\left(\begin{array}{ll}
1 & x \\
0 & 1
\end{array}\right) k\right) \log \overline{\mathrm{t}}\left(\left(\begin{array}{c}
1 \\
0 \\
0
\end{array} \frac{x}{1-\left(t_{2} / t_{1}\right)}\right)\right) d x d k \\
= & \operatorname{vol}\left(T(\mathbb{Q}) \backslash T(\mathbb{A})^{1}\right) \int_{\boldsymbol{K}} \int_{U(\mathbb{A})} f\left(k^{-1} t u k\right) \sum_{p \leq \infty} \omega_{p}^{\mathrm{std}}(t, u) d u d k .
\end{aligned}
$$

We choose $S$ such that for $t \in T(\mathbb{Q}) \backslash Z(\mathbb{Q})$ and $u \in U(\mathbb{A})$ we have $f(t u)=0$ unless $\left|\frac{t_{1}}{t_{2}}\right|_{p}=\left|1-\frac{t_{2}}{t_{1}}\right|_{p}=1$ and $u_{p} \in U\left(\mathbb{Z}_{p}\right)$ for all $p \notin S$, and in particular, $\lambda_{t, S}=0$ and $\omega(t, x)=\sum_{p \leq \infty} \omega_{p}^{\text {std }}(t, x)$. This is possible since there are only finitely many $t$ 's, depending on the support of $f$, which give a non-zero contribution. We can therefore write the above integral as

$$
\operatorname{vol}\left(T(\mathbb{Q}) \backslash T(\mathbb{A})^{1}\right) \int_{\boldsymbol{K}} \int_{U(\mathbb{A})} f\left(k^{-1} t u k\right) \omega(t, u) d u d k,
$$

which coincides with the expression in Theorem 1.

Similarly we can write the unipotent contribution (for $S$ sufficiently large) as

$$
\begin{aligned}
{\left[(s-1) \zeta^{S}(s) \int_{\mathbb{Q}_{S}^{*}} \Phi(x)|x|^{s} d^{*} x\right]_{s=1}^{\prime}=} & {\left[(s-1) \zeta^{S}(s)\right]_{s=1}^{\prime} \cdot \int_{\mathbb{Q}_{S}^{*}} \Phi(x)|x|_{S} d^{*} x } \\
& +\operatorname{res}_{s=1} \zeta^{S}(s) \cdot \int_{\mathbb{Q}_{S}^{*}} \Phi(x)|x|_{S} \log |x|_{S} d^{*} x .
\end{aligned}
$$

Converting the integration over $\mathbb{Q}_{S}^{*}$ to one over $\mathbb{Q}_{S}$ yields a factor of $\zeta_{S}(1)$. Using (4) we get

$$
\operatorname{res}_{s=1} \zeta^{*}(s) \lambda_{1, S} \int_{\mathbb{Q}_{S}} \Phi(x) d x+\operatorname{res}_{s=1} \zeta^{*}(s) \int_{\mathbb{Q}_{S}} \Phi(x) \log |x|_{S} d x .
$$

Once again, this coincides with the corresponding contribution of $t \in Z(\mathbb{Q})$ in Theorem 1.

Note that the rewriting of the unipotent contribution is especially important for the purpose of Theorem 1 since the Tate integral only makes sense for Schwartz-Bruhat functions.

A typical function which one may want to plug in the trace formula is

$$
f_{s}(g)=\int_{A_{G}} F_{S}(a g) d a
$$

where

$$
F_{S}(g)=e^{-\pi \operatorname{tr} g_{\infty}^{t} g_{\infty}} \chi_{M_{2}(\mathcal{O}) \cap G\left(\mathbb{A}_{\text {fin }}\right)}\left(g_{\text {fin }}\right)|\operatorname{det} g|^{s+\frac{1}{2}}
$$

for $\operatorname{Re}(s)>\frac{3}{2}$. On the spectral side, the contribution from the discrete spectrum is

$$
\sum_{\pi} L(s, \pi)
$$


where $\pi$ ranges over the representations occurring discretely in $L^{2}\left(A_{G} G(\mathbb{Q}) \backslash G(\mathbb{A})\right)$ which have a $\boldsymbol{K}$-fixed vector, and $L(s, \pi)$ is the (complete) standard $L$-function ([GJ72]). Note that by [Shi71], p. 64, and [GJ72]

$$
\int_{G(\mathbb{A})^{1}} f_{s}(g) d g=\int_{G(\mathbb{A})} F_{s}(g) d g=\zeta^{*}\left(s+\frac{1}{2}\right) \zeta^{*}\left(s-\frac{1}{2}\right)
$$

and both sides are absolutely convergent when $\operatorname{Re}(s)>\frac{3}{2}$. Moreover, for any $X, Y \in$ $u\left(\mathfrak{g}_{\mathbb{C}}^{1}\right)$,

$$
\left[X * F_{S} * Y\right](g)=P\left(g_{\infty}\right) F_{S}(g)
$$

for some polynomial $P=P_{X, Y}$ in the entries of $g_{\infty}$, as is easily seen by induction on the degrees of $X$ and $Y$. It follows once again from [GJ72] that

$$
\int_{G(\mathbb{A})^{1}}\left|X * f_{S} * Y(g)\right| d g=\int_{G(\mathbb{A})}\left|X * F_{S} * Y(g)\right| d g=\int_{G(\mathbb{A})}\left|P\left(g_{\infty}\right) F_{S}(g)\right| d g<\infty
$$

for $\operatorname{Re}(s)>\frac{3}{2}$, so that $f_{s} \in \mathcal{C}\left(G(\mathbb{A})^{1} ; \boldsymbol{K}_{\text {fin }}\right)$ in this region.

\section{Auxiliary estimates}

In this section we study some local integrals which appear in the analysis of the trace formula. In addition, we prove a simple but crucial lemma on lattice sums.

$p$-adic estimates. For $k, n \in \mathbb{Z}, n \geq 0$, let $f_{k, n}$ be the characteristic function of the subset

$$
\left(\begin{array}{cc}
p^{k} & 0 \\
0 & p^{k}
\end{array}\right) \boldsymbol{K}_{p}\left(\begin{array}{cc}
p^{n} & 0 \\
0 & 1
\end{array}\right) \boldsymbol{K}_{p}
$$

of $G\left(\mathbb{Q}_{p}\right)$. These functions form a basis for the Hecke algebra of bi- $\boldsymbol{K}_{p}$-invariant functions on this group. Recall that

$$
f_{k, n}\left(\left(\begin{array}{ll}
a & b \\
c & d
\end{array}\right)\right)=1 \Longleftrightarrow \max (|a|,|b|,|c|,|d|)=p^{-k} \text { and }\left|\operatorname{det}\left(\begin{array}{ll}
a & b \\
c & d
\end{array}\right)\right|=p^{-(2 k+n)} .
$$

(We often suppress the subscript $p$ from $|\cdot|$ if it is clear from the context.) Let $w=\left(\begin{array}{ll}0 & 1 \\ 1 & 0\end{array}\right)$.

Lemma 3.1. Let $t=\left(\begin{array}{cc}t_{1} & 0 \\ 0 & t_{2}\end{array}\right) \in T\left(\mathbb{Q}_{p}\right)$. The integral

$$
\int_{U\left(\mathbb{Q}_{p}\right)} \int_{U\left(\mathbb{Q}_{p}\right)} f_{k, n}\left(\left(\begin{array}{cc}
p^{k} & 0 \\
0 & p^{k}
\end{array}\right) t x w y\right) d x d y
$$

vanishes unless $|\operatorname{det} t|=p^{-n}$ and $\left|t_{2}\right| \leq 1$, in which case it is bounded by

$$
\left|t_{1}\right|^{-1}\left(\min \left(v_{p}\left(t_{2}\right), n\right)+1\right) \text {. }
$$


Proof. Without loss of generality we can assume that $k=0$. The condition on the determinant is clear. Let $m_{i}=v_{p}\left(t_{i}\right), i=1,2$. Since

$$
\left(\begin{array}{cc}
t_{1} & 0 \\
0 & t_{2}
\end{array}\right)\left(\begin{array}{ll}
1 & x \\
0 & 1
\end{array}\right) w\left(\begin{array}{ll}
1 & y \\
0 & 1
\end{array}\right)=\left(\begin{array}{cc}
t_{1} x & t_{1}(1+x y) \\
t_{2} & t_{2} y
\end{array}\right),
$$

the condition $m_{2} \geq 0$ follows as well. Moreover, the integral is bounded by the volume of the subset $C$ of $\mathbb{Q}_{p}^{2}$ defined by the inequalities

$$
|x| \leq p^{m_{1}}, \quad|1+x y| \leq p^{m_{1}}, \quad|y| \leq p^{m_{2}} .
$$

Note that each of the sets

$$
\begin{aligned}
& D_{i}=\left\{(x, y):|x|=p^{m_{1}-i},|1+x y| \leq p^{m_{1}}\right\}, \\
& E_{j}=\left\{(x, y):|y|=p^{m_{2}-j},|1+x y| \leq p^{m_{1}}\right\}
\end{aligned}
$$

has volume $\left(1-p^{-1}\right) p^{m_{1}}$ regardless of $i$ and $j$. The lemma follows from the easily verified inclusions

$$
C \subseteq \begin{cases}\left\{(x, y):|x| \leq p^{m_{1}-m_{2}},|y| \leq p^{m_{2}}\right\} \cup \bigcup_{0 \leq i<m_{2}} D_{i} & \text { for any } n, m_{1}, m_{2}, \\ \bigcup_{0 \leq j \leq n} E_{j} & \text { for } n<m_{2} .\end{cases}
$$

Lemma 3.2. Let $t=\left(\begin{array}{cc}t_{1} & 0 \\ 0 & t_{2}\end{array}\right) \in T\left(\mathbb{Q}_{p}\right)$. Then

$$
\int_{U\left(\mathbb{Q}_{p}\right)} f_{k, n}\left(\left(\begin{array}{cc}
p^{k} & 0 \\
0 & p^{k}
\end{array}\right) t u\right) d u=\int_{U\left(\mathbb{Q}_{p}\right)} f_{k, n}\left(\left(\begin{array}{cc}
p^{k} & 0 \\
0 & p^{k}
\end{array}\right) t u\right)\left|\omega_{p}(t, u)\right| d u=0,
$$

unless $|\operatorname{det} t|=p^{-n}$ and $\left|t_{1}\right|,\left|t_{2}\right| \leq 1$, in which case

$$
\begin{gathered}
\int_{U\left(\mathbb{Q}_{p}\right)} f_{k, n}\left(\left(\begin{array}{cc}
p^{k} & 0 \\
0 & p^{k}
\end{array}\right) t u\right) d u \leq\left|t_{1}\right|^{-1}, \\
\int_{U\left(\mathbb{Q}_{p}\right)} f_{k, n}\left(\left(\begin{array}{cc}
p^{k} & 0 \\
0 & p^{k}
\end{array}\right) t u\right)\left|\omega_{p}(t, u)\right| d u \leq\left|t_{1}\right|^{-1}\left(-\log \left|t_{1}\right|+\delta_{p}\right),
\end{gathered}
$$

where $\delta_{p}=1$ if $p \in S$ and 0 otherwise.

Proof. We can assume that $k=0$. The condition on the determinant is clear. Assume from now on that $|\operatorname{det} t|=p^{-n}$. Note that $f_{0, n}\left(\left(\begin{array}{ccc}t_{1} & 0 \\ 0 & t_{2}\end{array}\right)\left(\begin{array}{ll}1 & x \\ 0 & 1\end{array}\right)\right)=1$ iff

$$
\max \left(\left|t_{1}\right|,\left|t_{1} x\right|,\left|t_{2}\right|\right)=1 .
$$

The vanishing statement and (13) follow easily. To show (14) we separate into cases. Suppose first that $\left|t_{1}\right| \neq\left|t_{2}\right|$. Then

$$
0 \leq \omega_{p}(t, u) \leq-\log \left|t_{1}\right| \quad \text { whenever } f_{0, n}(t u)=1 .
$$


Thus (14) follows. Suppose now that $\left|t_{1}\right|=\left|t_{2}\right|$. If $p \in S$ and $\left|t_{1}\right|=\left|t_{2}\right|<1$ then once again (15) holds since $|x|=\left|t_{1}\right|^{-1}>1$ where $u=\left(\begin{array}{ll}1 & x \\ 0 & 1\end{array}\right)$. On the other hand, if $p \in S$ and $\left|t_{1}\right|=\left|t_{2}\right|=1$ then the integral is bounded by

$$
\sum_{k=0}^{\infty} k p^{-k}\left(1-p^{-1}\right) \log p=\frac{\log p}{p-1} \leq 1 .
$$

Finally if $p \notin S$ and $\left|t_{1}\right|=\left|t_{2}\right|$ then the integral is 0 unless $\left|t_{1}\right|<1$ in which case it is bounded by

$$
\left|t_{1}\right|^{-1}\left(1-p^{-1}\right)\left(-\log \left|t_{1}\right|+\frac{\log p}{p-1}\right) \leq-\left|t_{1}\right|^{-1} \log \left|t_{1}\right|
$$

as required.

Remark 1. For any $p \notin S$ and $k \in \mathbb{Z}$ we have

$$
\int_{U\left(\mathbb{Q}_{p}\right)} f_{k, 0}(t u) \omega_{p}^{\mathrm{std}}(t, u) d u= \begin{cases}-\frac{1-\left|1-\frac{t_{2}}{t_{1}}\right|_{p}}{p-1} \log p, & \text { if }\left|t_{1}\right|_{p}=\left|t_{2}\right|_{p}=p^{-k}, \\ 0, & \text { otherwise, }\end{cases}
$$

as a consequence of the identity

$$
p^{-m} \log p^{-m}+\sum_{j=0}^{m-1} p^{-j}\left(1-p^{-1}\right) \log \left(p^{-j}\right)=-\frac{1-\left|1-\frac{t_{2}}{t_{1}}\right|}{p-1} \log p
$$

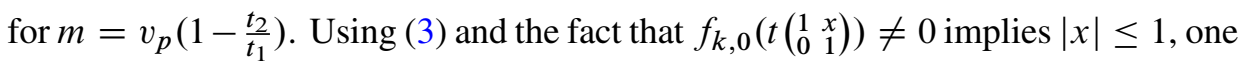
obtains from this the local identity (9) for the functions $f_{k, 0}$. For the functions $f_{k, n}$, $n>0$, this identity is an immediate consequence of the definitions together with the observation that $f_{k, n}\left(t\left(\begin{array}{ll}1 & x \\ 0 & 1\end{array}\right)\right)$ can be only non-zero for $|x|>1$ if $n>0$. It follows that (9) is true in general and that the geometric side of the identity in Theorem 1 is invariant under enlarging $S$.

Recall that the set $\left\{\left(\begin{array}{ll}r & 0 \\ 0 & r\end{array}\right)\left(\begin{array}{ll}N & 0 \\ 0 & 1\end{array}\right): r \in \mathbb{Q}_{>0}, N \in \mathbb{N}\right\}$ forms a set of representatives for $\boldsymbol{K}_{\text {fin }} \backslash G\left(\mathbb{A}_{\text {fin }}\right) / \boldsymbol{K}_{\text {fin }}$. Let $T_{r, N}, r \in \mathbb{Q}>0, N \geq 1$, denote the characteristic function of the double coset

$$
\left(\begin{array}{ll}
r & 0 \\
0 & r
\end{array}\right) \boldsymbol{K}_{\text {fin }}\left(\begin{array}{ll}
N & 0 \\
0 & 1
\end{array}\right) \boldsymbol{K}_{\text {fin }}
$$

in $G\left(\mathbb{A}_{\text {fin }}\right)$. Recall that

$$
\operatorname{deg} T_{r, N}:=\int_{G\left(\mathbb{A}_{\text {fin }}\right)} T_{r, N}(x) d x=N \prod_{p \mid N}\left(1+p^{-1}\right) .
$$

As usual, denote by $\sigma_{s}$ the divisor function

$$
\sigma_{s}(n)=\sum_{d \mid n} d^{s}
$$

From the estimates above we immediately obtain 
Proposition 3.3. (1) Let $t \in \mathbb{Q}^{*}$. Then

$$
\int_{U\left(\mathbb{A}_{\mathrm{fin}}\right)} \int_{U\left(\mathbb{A}_{\mathrm{fin}}\right)} T_{r, N}\left(\left(\begin{array}{cc}
r & 0 \\
0 & r
\end{array}\right)\left(\begin{array}{cc}
N t^{-1} & 0 \\
0 & t
\end{array}\right) x w y\right) d x d y=0
$$

unless $t \in \mathbb{Z}$, in which case it is bounded by $\frac{N}{|t|} \sigma_{0}(\operatorname{gcd}(N,|t|))$.

(2) For $t=\left(\begin{array}{cc}t_{1} & 0 \\ 0 & t_{2}\end{array}\right) \in T(\mathbb{Q})$ the integrals

$$
\int_{U\left(\mathbb{A}_{\mathrm{fin}}\right)} T_{r, N}\left(\left(\begin{array}{cc}
r & 0 \\
0 & r
\end{array}\right) t u\right) d u, \quad \int_{U\left(\mathbb{A}_{\mathrm{fin}}\right)} T_{r, N}\left(\left(\begin{array}{cc}
r & 0 \\
0 & r
\end{array}\right) t u\right) \sum_{p<\infty}\left|\omega_{p}(t, u)\right| d u
$$

vanish unless det $t= \pm N$ and $t_{1}, t_{2} \in \mathbb{Z}$, in which case the first is bounded by $\left|t_{1}\right|$, and the second by $\left|t_{1}\right|\left(\log \left|t_{1}\right|+|S|-1\right)$.

Lattice sums. For any absolutely continuous function $f$ on $\mathbb{R}$ such that $f, f^{\prime} \in$ $L^{1}(\mathbb{R})$ we have

$$
\sum_{n \in \mathbb{Z}}|f(n)| \leq\|f\|_{1}+\left\|f^{\prime}\right\|_{1}
$$

Indeed, for any $0 \leq x \leq 1$

$$
\sum_{n \in \mathbb{Z}}|f(n)-f(n+x)| \leq \sum_{n \in \mathbb{Z}} \int_{n}^{n+x}\left|f^{\prime}(t)\right| d t \leq\left\|f^{\prime}\right\|_{1},
$$

so that

$$
\sum_{n \in \mathbb{Z}}|f(n)| \leq\left\|f^{\prime}\right\|_{1}+\sum_{n \in \mathbb{Z}}|f(n+x)| .
$$

Integrating over $x$ we obtain (16).

For an open subgroup $\mathcal{O}^{\prime}$ of $\mathcal{O}$ we denote by $\mathcal{C}\left(\mathbb{A} ; \mathcal{O}^{\prime}\right)$ the space of smooth $\mathcal{O}^{\prime}$ invariant functions $f$ on $\mathbb{A}$ such that $f^{(n)} \in L^{1}\left(\mathbb{A} / \mathcal{O}^{\prime}\right), n=0,1, \ldots$

Lemma 3.4. For any $f \in \mathcal{C}\left(\mathbb{A} ; \mathcal{O}^{\prime}\right)$ we have

$$
\sum_{\gamma \in \mathbb{Q}}|f(\gamma)| \leq\left[\mathcal{O}: \mathcal{O}^{\prime}\right]\left(\|f\|_{1}+\left\|f^{\prime}\right\|_{1}\right) .
$$

Proof. For each $r \in \mathcal{O}^{\prime} \backslash \mathbb{A}_{\text {fin }}$ let $f_{r}$ be the restriction of $f$ to $\mathbb{R}+r+\mathcal{O}^{\prime}$. Thus, we can write $f=\sum_{r \in \mathcal{O}^{\prime} \backslash \mathbb{A}_{\mathrm{fin}}} f_{r}$ where $f_{r}(x)=g_{r}\left(x_{\infty}\right) \chi_{r+\mathcal{O}^{\prime}}\left(x_{\mathrm{fin}}\right)$ and $g_{r}\left(x_{\infty}\right)$ is the value of $f$ on the coset $x_{\infty}+r+\mathcal{O}^{\prime}$. By (16), for any $r$

$$
\sum_{n \in \mathbb{Q} \cap \mathcal{O}^{\prime}}\left|g_{r}(n+r)\right| \leq \sum_{n \in \mathbb{Z}}\left|g_{r}(n+r)\right| \leq\left\|g_{r}\right\|_{1}+\left\|g_{r}^{\prime}\right\|_{1} .
$$


Summing over $r \in \mathcal{O}^{\prime} \backslash \mathbb{A}_{\text {fin }}$ we obtain

$$
\begin{aligned}
\sum_{\gamma \in \mathbb{Q}}|f(\gamma)| & =\sum_{r \in \mathcal{O}^{\prime} \backslash \mathbb{A}_{\text {fin }}} \sum_{n \in \mathbb{Q} \cap \mathcal{O}^{\prime}}\left|g_{r}(n+r)\right| \\
& \leq \sum_{r \in \mathcal{O}^{\prime} \backslash \mathbb{A}_{\text {fin }}}\left(\left\|g_{r}\right\|_{1}+\left\|g_{r}^{\prime}\right\|_{1}\right)=\left[\mathcal{O}: \mathcal{O}^{\prime}\right]\left(\|f\|_{1}+\left\|f^{\prime}\right\|_{1}\right),
\end{aligned}
$$

as required.

Archimedean estimates. Let $G(\mathbb{R})^{1}=G(\mathbb{R}) \cap G(\mathbb{A})^{1}=\{g \in G(\mathbb{R}):|\operatorname{det} g|=$ $1\}$ and $T(\mathbb{R})^{1}=T(\mathbb{R}) \cap G(\mathbb{R})^{1}$. We have

$$
G(\mathbb{R}) \simeq G(\mathbb{R})^{1} \times A_{G}, \quad T(\mathbb{R}) \simeq T(\mathbb{R})^{1} \times A_{G} .
$$

We endow $G(\mathbb{R})^{1}$ and $T(\mathbb{R})^{1}$ with Haar measures accordingly.

We denote by $\ell\left(G(\mathbb{R})^{1}\right)$ the Fréchet algebra of smooth functions on $G(\mathbb{R})^{1}$ such that $X * f * Y \in L^{1}\left(G(\mathbb{R})^{1}\right)$ for all $X, Y \in \mathcal{U}\left(g_{\mathbb{C}}^{1}\right)$ with respect to the norms

$$
\sum_{i, j \geq 0, i+j \leq n} \sum_{X \in \mathcal{B}_{i}, Y \in \mathcal{B}_{j}}\|X * f * Y\|_{1}, \quad n=0,1, \ldots
$$

By a standard integration formula with respect to the Cartan decomposition, the integral of a function $f \in L^{1}\left(G(\mathbb{R})^{1}\right)$ can be expressed as

$$
\int_{G(\mathbb{R})^{1}} f(g) d g=8 \pi \int_{\boldsymbol{K}_{\infty}} \int_{1}^{\infty} \int_{\boldsymbol{K}_{\infty}} f\left(k_{1}\left(\begin{array}{cc}
a & 0 \\
0 & a^{-1}
\end{array}\right) k_{2}\right)\left(a^{2}-a^{-2}\right) d k_{1} d^{*} a d k_{2} .
$$

Another useful integration formula is

$$
\int_{T(\mathbb{R})^{1}} \int_{U(\mathbb{R})} \int_{U(\mathbb{R})} f(t x w y) d x d y d t=\pi \int_{G(\mathbb{R})^{1}} f(g) d g .
$$

Identify the Lie algebra $g$ of $G$ with the algebra of $2 \times 2$ matrices. Let $H$ be any of the one-dimensional Lie groups $T(\mathbb{R})^{1}, U(\mathbb{R}), \bar{U}(\mathbb{R})$ or $\boldsymbol{K}_{\infty}$. To each $H$ we attach a generator $x_{H}$ of its Lie algebra. Explicitly, $x_{H}=\left(\begin{array}{cc}1 & 0 \\ 0 & -1\end{array}\right),\left(\begin{array}{ll}0 & 1 \\ 0 & 0\end{array}\right),\left(\begin{array}{ll}0 & 0 \\ 1 & 0\end{array}\right)$, or $\left(\begin{array}{cc}0 & 1 \\ -1 & 0\end{array}\right)$, respectively. As in the argument proving (16) we have

$$
\begin{aligned}
& \sup _{h \in H}|f(h g)| \leq \int_{H}|f(h g)| d h+\int_{H}\left|x_{H} * f(h g)\right| d h, \\
& \sup _{h \in H}|f(g h)| \leq \int_{H}|f(g h)| d h+\int_{H}\left|f * x_{H}(g h)\right| d h,
\end{aligned}
$$

for all $g \in G(\mathbb{R})^{1}$. It follows that

$$
\tilde{f}(g):=\max _{k_{1}, k_{2} \in \boldsymbol{K}_{\infty}}\left|f\left(k_{1} g k_{2}\right)\right| \leq \sum_{Y_{1}, Y_{2}=1, x_{\boldsymbol{K}}} \int_{\boldsymbol{K}_{\infty}} \int_{\boldsymbol{K}_{\infty}}\left|Y_{1} * f * Y_{2}\left(k_{1} g k_{2}\right)\right| d k_{1} d k_{2} .
$$


Using the integration formula (17) we infer that

$$
\begin{aligned}
\int_{1}^{\infty} & \tilde{f}\left(\left(\begin{array}{ll}
a & 0 \\
0 & a^{-1}
\end{array}\right)\right)\left(a^{2}-a^{-2}\right) d^{*} a \\
& \leq \sum_{\substack { Y_{1}, Y_{2}=1 \\
\begin{subarray}{c}{x \\
\boldsymbol{K}_{\infty}{ Y _ { 1 } , Y _ { 2 } = 1 \\
\begin{subarray} { c } { x \\
\boldsymbol { K } _ { \infty } } }\end{subarray}} \int_{1}^{\infty} \int_{\substack{k_{1}, k_{2} \in \boldsymbol{K}_{\infty}\\
}}\left|Y_{1} * f * Y_{2}\left(k_{1}\left(\begin{array}{cc}
a & 0 \\
0 & a^{-1}
\end{array}\right) k_{2}\right)\right|\left(a^{2}-a^{-2}\right) d k_{1} d k_{2} d^{*} a \\
& =(8 \pi)^{-1} \sum_{Y_{1}, Y_{2}=1, x_{K_{\infty}}}\left\|Y_{1} * f * Y_{2}\right\|_{1}
\end{aligned}
$$

Lemma 3.5. The norm $\sup _{g \in G(\mathbb{R})^{1}}|f(g)|$ is continuous on $\mathcal{C}\left(G(\mathbb{R})^{1}\right)$.

Proof. Consider the inequality

$$
|\varphi(0)| \leq \sum_{I \subseteq\{1,2,3\}} \int_{[0,1]^{3}}\left|\frac{\partial^{|I|} \varphi}{\prod_{i \in I} \partial x_{i}}(x)\right| d x
$$

for any smooth function $\varphi$ on $[0,1]^{3}$, which is valid by the Fundamental Theorem of Calculus.

Using local coordinates, it follows that for any compact neighborhood $C$ of the identity in $G(\mathbb{R})^{1}$ there exists a constant $c>0$ with

$$
|f(e)| \leq c \sum_{0 \leq i \leq 3, X \in B_{i}} \int_{C}|f * X(g)| d g
$$

for all $f \in \ell\left(G(\mathbb{R})^{1}\right)$, which clearly implies

$$
|f(e)| \leq c \sum_{0 \leq i \leq 3, X \in \mathcal{B}_{i}}\|f * X\|_{1} .
$$

Applying left translation by arbitrary elements of $G(\mathbb{R})^{1}$ finishes the proof.

Lemma 3.6. There exists an absolute constant $C>0$ such that for all $t=\left(\begin{array}{cc}t_{1} & 0 \\ 0 & t_{2}\end{array}\right) \in$ $T(\mathbb{R})^{1}$ and $u=\left(\begin{array}{ll}1 & x \\ 0 & 1\end{array}\right) \in U(\mathbb{R})$ with $|x| \geq\left|\frac{t_{2}}{t_{1}}\right|$ we have

$$
\left|\omega_{\infty}(t, u)\right| \leq C \min \left(|x|,\left|\frac{t_{1}}{t_{2}} x\right|\right) \log \left(2+\left|\frac{t_{2}}{t_{1}}\right|\right) .
$$

Proof. This is straightforward. We can assume that $x \geq 0$. Consider first the case $\left|\frac{t_{2}}{t_{1}}\right| \geq \frac{1}{2}$. Clearly, $\omega_{\infty}(t, u)$ is then bounded from below. To show the upper bound note that

$$
\left(1-\frac{t_{2}}{t_{1}}\right)^{2} \leq(1+x)^{2} \leq 9 x^{2}
$$

and therefore

$$
\omega_{\infty}(t, u) \leq \log x+\frac{\log 10}{2}
$$


The inequality (21) follows now from the fact that $\log x / x$ is monotone decreasing for $x \geq e$.

Now assume that $\left|\frac{t_{2}}{t_{1}}\right| \leq \frac{1}{2}$. Then $\omega_{\infty}\left(t,\left(\begin{array}{ll}1 & x \\ 0 & 1\end{array}\right)\right)-x$ is monotone decreasing for $x \geq 0$ since

$$
\frac{x}{\left(1-\frac{t_{2}}{t_{1}}\right)^{2}+x^{2}} \leq \frac{x}{\frac{1}{4}+x^{2}} \leq 1
$$

Thus, $\omega_{\infty}\left(t,\left(\begin{array}{ll}1 & x \\ 0 & 1\end{array}\right)\right) \leq x$ for $x \geq\left|\frac{t_{2}}{t_{1}}\right|$ since

$$
\frac{1}{2} \log \left(\left(1-\frac{t_{2}}{t_{1}}\right)^{2}+\left|\frac{t_{2}}{t_{1}}\right|^{2}\right) \leq\left|\frac{t_{2}}{t_{1}}\right|
$$

from the Taylor expansion of the exponential function. Also, for $x \geq\left|\frac{t_{2}}{t_{1}}\right|$,

$$
\omega_{\infty}\left(t,\left(\begin{array}{ll}
1 & x \\
0 & 1
\end{array}\right)\right) \geq \frac{1}{2} \log \left(\left(1-\frac{t_{2}}{t_{1}}\right)^{2}+\left|\frac{t_{2}}{t_{1}}\right|^{2}\right) \geq-\left|\frac{t_{2}}{t_{1}}\right| .
$$

Thus, (21) holds in this case as well.

Lemma 3.7. There exists a continuous seminorm $\mu$ on $\mathcal{C}\left(G(\mathbb{R})^{1}\right)$ such that

$$
\int_{U(\mathbb{R})}|f(t u)| d u \leq \frac{\mu(f)}{\max \left(1,\left|\frac{t_{1}}{t_{2}}\right|\right)}
$$

and

$$
\int_{U(\mathbb{R})}\left|f(t u) \omega_{\infty}(t, u)\right| d u \leq \frac{\mu(f) \log \left(2+\left|\frac{t_{2}}{t_{1}}\right|\right)}{\max \left(1,\left|\frac{t_{1}}{t_{2}}\right|\right)}
$$

for all $t=\left(\begin{array}{cc}t_{1} & 0 \\ 0 & t_{2}\end{array}\right) \in T(\mathbb{R})^{1}$ and $f \in \mathcal{C}\left(G(\mathbb{R})^{1}\right)$.

Proof. Using (19) we get

$$
\sup _{k \in \boldsymbol{K}_{\infty}, t \in T(\mathbb{R})^{1}}|f(\operatorname{tg} k)| \leq \sum_{X_{1}=1, x_{T(\mathbb{R})^{1}}} \sum_{X_{2}=1, x_{\boldsymbol{K}}} \int_{\boldsymbol{K}_{\infty}} \int_{T(\mathbb{R})^{1}}\left|X_{1} * f * X_{2}(\operatorname{tg} k)\right| d t d k .
$$

Integrating over $g \in U(\mathbb{R})$ we get

$$
\begin{aligned}
\sup _{t \in T(\mathbb{R})^{1}} & \int_{U(\mathbb{R})}|f(t u)| d u \\
\leq & \sum_{X_{1}=1, x_{T(\mathbb{R})^{1}}} \sum_{X_{2}=1, x_{K_{\infty}}} \int_{\boldsymbol{K}_{\infty}} \int_{U(\mathbb{R})} \int_{T(\mathbb{R})^{1}}\left|X_{1} * f * X_{2}(t u k)\right| d t d u d k \\
= & \sum_{X_{1}=1, x_{T(\mathbb{R})^{1}}} \sum_{X_{2}=1, x_{K_{\infty}}}\left\|X_{1} * f * X_{2}\right\|_{1} .
\end{aligned}
$$


In a similar vein,

$$
\begin{aligned}
\sup _{t \in T(\mathbb{R})^{1}} & \int_{U(\mathbb{R})}|f(u t)| d u \\
\leq & \sum_{X_{1}=1, x_{K_{\infty}}} \sum_{X_{2}=1, x_{T(\mathbb{R}) 1}} \int_{\boldsymbol{K}_{\infty}} \int_{U(\mathbb{R})} \int_{T(\mathbb{R})^{1}}\left|X_{1} * f * X_{2}(k u t)\right| d t d u d k \\
= & \sum_{X_{1}=1, x_{K_{\infty}}} \sum_{X_{2}=1, x_{T(\mathbb{R})^{1}}}\left\|X_{1} * f * X_{2}\right\|_{1} .
\end{aligned}
$$

Combining the two we obtain (22). To show (23) we write

$$
\begin{aligned}
& \int_{U(\mathbb{R})}\left|f(t u) \omega_{\infty}(t, u)\right| d u=\int_{|x| \leq \max \left(1,\left|\frac{t_{2}}{t_{1}}\right|\right)}\left|f\left(\left(\begin{array}{cc}
t_{1} & t_{1} x \\
0 & t_{2}
\end{array}\right)\right) \omega_{\infty}\left(t,\left(\begin{array}{ll}
1 & x \\
0 & 1
\end{array}\right)\right)\right| d x \\
& +\int_{|x| \geq \max \left(1,\left|\frac{t_{2}}{t_{1}}\right|\right)}\left|f\left(\left(\begin{array}{cc}
t_{1} & t_{1} x \\
0 & t_{2}
\end{array}\right)\right) \omega_{\infty}\left(t,\left(\begin{array}{ll}
1 & x \\
0 & 1
\end{array}\right)\right)\right| d x \text {. }
\end{aligned}
$$

To deal with the first integral, we split into two cases. If $\left|1-\frac{t_{2}}{t_{1}}\right| \geq \frac{1}{2}$, we bound it by a constant multiple of

$$
\log \left(2+\left|\frac{t_{2}}{t_{1}}\right|\right) \int_{\mathbb{R}}\left|f\left(\left(\begin{array}{cc}
t_{1} & t_{1} x \\
0 & t_{2}
\end{array}\right)\right)\right| d x
$$

which we already know how to bound. If $\left|1-\frac{t_{2}}{t_{1}}\right| \leq \frac{1}{2}$, we bound the integral by a constant multiple of

$$
\int_{0}^{3 / 2}(1+|\log x|) d x \sup _{\substack{\frac{1}{\sqrt{2}} \leq\left|t_{1}\right|,\left|t_{2}\right| \leq \sqrt{2} \\
|x| \leq \frac{3}{2}}}\left|f\left(\left(\begin{array}{cc}
t_{1} & t_{1} x \\
0 & t_{2}
\end{array}\right)\right)\right|
$$

which is a continuous seminorm. To deal with the second integral in (24) write

$$
\left(\begin{array}{cc}
t_{1} & t_{1} x \\
0 & t_{2}
\end{array}\right)=k_{1}\left(\begin{array}{cc}
a & 0 \\
0 & a^{-1}
\end{array}\right) k_{2}, \quad k_{1}, k_{2} \in \boldsymbol{K}_{\infty},
$$

where $a=a(x)>1$ satisfies $a^{2}+a^{-2}=t_{1}^{2}\left(1+x^{2}\right)+t_{2}^{2}$. Thus

$$
\frac{a^{\prime}(x)}{a(x)}\left(a(x)^{2}-a(x)^{-2}\right)=t_{1}^{2} x .
$$

By Lemma 3.6 and (20), the second integral in (24) is bounded by a constant multiple 
of

$$
\begin{aligned}
& \frac{\log \left(2+\left|\frac{t_{2}}{t_{1}}\right|\right)}{\max \left(1,\left|\frac{t_{1}}{t_{2}}\right|\right)} \int_{|x| \geq \max \left(1,\left|\frac{t_{2}}{t_{1}}\right|\right)} \tilde{f}\left(\left(\begin{array}{cc}
a(x) & 0 \\
0 & a(x)^{-1}
\end{array}\right)\right) \frac{a(x)^{2}-a(x)^{-2}}{a(x)} a^{\prime}(x) d x \\
& \quad \leq \frac{\log \left(2+\left|\frac{t_{2}}{t_{1}}\right|\right)}{\max \left(1,\left|\frac{t_{1}}{t_{2}}\right|\right)} \int_{1}^{\infty} \tilde{f}\left(\left(\begin{array}{cc}
a & 0 \\
0 & a^{-1}
\end{array}\right)\right)\left(a^{2}-a^{-2}\right) d^{*} a \\
& \leq(8 \pi)^{-1} \frac{\log \left(2+\left|\frac{t_{2}}{t_{1}}\right|\right)}{\max \left(1,\left|\frac{t_{1}}{t_{2}}\right|\right)} \sum_{Y_{1}=1, x_{K_{\infty}}} \sum_{Y_{2}=1, x_{K} K_{\infty}}\left\|Y_{1} * f * Y_{2}\right\|_{1} .
\end{aligned}
$$

\section{Continuity of the trace formula}

We are now ready to show the continuity of the seminorms (5)-(8). Note that each distribution is invariant under conjugation by $\boldsymbol{K}$. By passing to $\int_{\boldsymbol{K}} f\left(k^{-1} \cdot k\right) d k$ we can assume at the outset that $f$ is invariant under conjugation by $\boldsymbol{K}$.

The spectral side is easy to handle. The fact that the trace norm of $R_{\text {disc }}(f)$ is a continuous seminorm follows from the simplest bounds on the number of cuspidal automorphic representations on $G(\mathbb{Q}) \backslash G(\mathbb{A})$ with a $K$-fixed vector as the infinitesimal character grows. (Any polynomial bound will suffice.) Furthermore, there are only finitely many Dirichlet characters (depending on $K$ ) contributing to the continuous part, and the contribution of each one can be easily majorized. We omit the details, since the continuity of (8) was proved in much greater generality in [FLM].

Consider the geometric side. We first make a simple reduction. For any $x \in$ $G\left(\mathbb{A}_{\text {fin }}\right)$ let $f_{x} \in \mathcal{C}\left(G(\mathbb{A})^{1} ; K\right)$ be the restriction of $f$ to the inverse image of the coset $K x K$ under the map $g \mapsto g_{\text {fin }}$. Then

$$
f=\sum_{x \in K \backslash G\left(\mathbb{A}_{\text {fin }}\right) / K} f_{x}
$$

and for any $X, Y \in \mathcal{U}\left(\mathrm{g}_{\mathbb{C}}^{1}\right)$

$$
\|X * f * Y\|_{1}=\sum_{x \in K \backslash G\left(\mathbb{A}_{\mathrm{fin}}\right) / K}\left\|(X * f * Y)_{x}\right\|_{1}=\sum_{x \in K \backslash G\left(\mathbb{A}_{\mathrm{fin}}\right) / K}\left\|X * f_{x} * Y\right\|_{1} .
$$

Thus, in proving the continuity of (5), (6) and (7) it suffices to show that these seminorms are bounded by $\mu(f)$ for some continuous seminorm $\mu$, whenever $f=$ $f_{x}$ for some $x \in K \backslash G\left(\mathbb{A}_{\text {fin }}\right) / K$. Fix $x$ and let $x^{1}$ be the $G(\mathbb{A})^{1}$-component of $x$ under the isomorphism (1). Define $f_{x}^{\infty} \in \mathcal{C}\left(G(\mathbb{R})^{1}\right)$ by $f_{x}^{\infty}(g)=f_{x}\left(x^{1} g\right)$. Thus, $f_{x}(g)=f_{x}^{\infty}\left(g_{\infty}^{1}\right) \chi_{K x K}\left(g_{\text {fin }}\right)$. Let $r \in \mathbb{Q}>0$ and $N \geq 1$ be such that $x \in$ $\left(\begin{array}{ll}r & 0 \\ 0 & r\end{array}\right) \boldsymbol{K}_{\text {fin }}\left(\begin{array}{cc}N & 0 \\ 0 & 1\end{array}\right) \boldsymbol{K}_{\mathrm{fin}}$. Then for any $X, Y \in \mathcal{U}\left(\mathfrak{g}_{\mathbb{C}}^{1}\right)$ we have

$$
\left\|X * f_{x} * Y\right\|_{1} \geq\left[\boldsymbol{K}_{\mathrm{fin}}: K\right]^{-2}\left\|X * f_{x}^{\infty} * Y\right\|_{1} \operatorname{deg}\left(T_{r, N}\right) .
$$


Thus, it suffices to show that there exists a continuous seminorm $\mu$ on $\mathcal{C}\left(G(\mathbb{R})^{1}\right)$ such that for all $r \in \mathbb{Q}>0, N \geq 1$ and $f_{\infty} \in \mathscr{C}\left(G(\mathbb{R})^{1}\right)$ each of the expressions (5), (6) and (7) for the function

$$
f(g)=f_{\infty}\left(g_{\infty}^{1}\right) T_{r, N}\left(g_{\text {fin }}\right) \in \mathcal{C}\left(G(\mathbb{A})^{1} ; \boldsymbol{K}_{\text {fin }}\right)
$$

is bounded by $\mu\left(f_{\infty}\right) \operatorname{deg} T_{r, N}$. We will consider each type of conjugacy class separately.

Central contribution. The sum $\sum_{z \in Z(\mathbb{Q})}|f(z)|$ for $f$ of the form (27) reduces to $f_{\infty}(e)+f_{\infty}(-e)$ since only $z= \pm\left(\begin{array}{ll}r & 0 \\ 0 & r\end{array}\right)$ contribute. This case immediately follows from Lemma 3.5.

Elliptic contribution. To deal with (5) we show that

$$
\int_{G(\mathbb{Q}) \backslash G(\mathbb{A})^{1}} \sum_{\gamma \in G(\mathbb{Q})_{\text {ell. reg. }}}\left|f\left(x^{-1} \gamma x\right)\right| d x
$$

is a continuous seminorm on $\mathcal{C}\left(G(\mathbb{A})^{1} ; K\right)$. Note that we can restrict to the regular elliptic elements, since the non-regular ones are the central elements.

For $c>0$ let

$$
\delta_{c}=\left\{u t k: u \in U(\mathbb{A}), k \in K, t \in T(\mathbb{A}) \cap G(\mathbb{A})^{1},\left|\frac{t_{1}}{t_{2}}\right|>c\right\} .
$$

Note that $S_{c}$ is left $B(\mathbb{Q})$-invariant. By reduction theory it is known that $G(\mathbb{Q}) S_{c}=$ $G(\mathbb{A})^{1}$ for $c \ll 1$.

Since $G(\mathbb{Q})_{\text {ell. reg. }} \subseteq B(\mathbb{Q}) w U(\mathbb{Q})$ it will suffice to show that

$$
\int_{B(\mathbb{Q}) \backslash s_{c}} \sum_{t \in T(\mathbb{Q})} \sum_{u, u^{\prime} \in U(\mathbb{Q})}\left|f\left(x^{-1} u^{\prime} t w u x\right)\right| d x
$$

is a continuous seminorm on $\mathcal{C}\left(G(\mathbb{A})^{1} ; K\right)$. Since $f$ is invariant under conjugation by $\boldsymbol{K}$ we write the integral as

$$
\begin{aligned}
\int_{U(\mathbb{Q}) \backslash U(\mathbb{A})} \int_{T(\mathbb{Q}) \backslash T(\mathbb{A})^{1}} \int_{\sqrt{c}}^{\infty} \sum_{t \in T(\mathbb{Q})} \sum_{u, u^{\prime} \in U(\mathbb{Q})} & \left|f\left(\left(\begin{array}{cc}
a^{-1} & 0 \\
0 & a
\end{array}\right) s^{-1} x^{-1} u^{\prime} t w u x s\left(\begin{array}{cc}
a & 0 \\
0 & a^{-1}
\end{array}\right)\right)\right| \frac{d^{*} a}{a^{2}} d s d x .
\end{aligned}
$$

Combining $u^{\prime}$ and $x$ and recalling that $\mathbb{I}^{1}=\mathbb{Q}^{*} \mathcal{O}^{*}$ where $\mathcal{O}^{*}=\prod_{p<\infty} \mathbb{Z}_{p}^{*}$, we can 
write this as

$$
\begin{aligned}
\int_{U(\mathbb{A})} \int_{\left(\mathbb{Q}^{*} \backslash \mathbb{I}^{1}\right)^{2}} & \int_{\sqrt{c}}^{\infty} \sum_{t \in T(\mathbb{Q})} \sum_{u \in U(\mathbb{Q})} \\
= & \left.\left.\int_{\mathbb{A}} \int_{\left(\mathcal{O}^{*}\right)^{2}} \int_{\sqrt{c}} \sum_{t \in T(\mathbb{Q})} \sum_{u \in \mathbb{Q}} \begin{array}{cc}
\left(a s_{1}\right)^{-1} & 0 \\
0 & a s_{2}
\end{array}\right) x^{-1} t w u x\left(\begin{array}{cc}
a s_{1} & 0 \\
0 & \left(a s_{2}\right)^{-1}
\end{array}\right)\right) \mid \frac{d^{*} a}{a^{2}} d s_{1} d s_{2} d x \\
& \left|f\left(\left(\begin{array}{cc}
\left(a s_{1}\right)^{-1} & 0 \\
0 & a s_{2}
\end{array}\right)\left(\begin{array}{cc}
1 & -x \\
0 & 1
\end{array}\right) t w\left(\begin{array}{cc}
a s_{1} & 0 \\
0 & \left(a s_{2}\right)^{-1}
\end{array}\right)\left(\begin{array}{cc}
1 & \frac{u+x}{a^{2} s_{1} s_{2}} \\
0 & 1
\end{array}\right)\right)\right| \frac{d^{*} a}{a^{2}} d s_{1} d s_{2} d x .
\end{aligned}
$$

Applying Lemma 3.4 we may replace the sum over $u$ by the sum of two integrals (of the functions $f$ and $a^{-2} f * x_{U(\mathbb{R})}$ ) over $\mathbb{A}$. Since $a$ is bounded from below, we reduce after a change of variables to the estimation of

$$
\int_{U(\mathbb{A})^{2}} \int_{\left(\mathcal{O}^{*}\right)^{2}} \int_{\sqrt{c}}^{\infty} \sum_{t \in T(\mathbb{Q})}\left|f\left(\left(\begin{array}{cc}
\left(a s_{1}\right)^{-1} & 0 \\
0 & a s_{2}
\end{array}\right) x t w\left(\begin{array}{cc}
a s_{1} & 0 \\
0 & \left(a s_{2}\right)^{-1}
\end{array}\right) y\right)\right| d^{*} a d s_{1} d s_{2} d x d y
$$

for $f$ and $f * x_{U(\mathbb{R})}$. Conjugating $\left(\begin{array}{cc}a s_{1} & 0 \\ 0 & \left(a s_{2}\right)^{-1}\end{array}\right)$ we can rewrite this as

$$
\begin{aligned}
& \int_{U(\mathbb{A})^{2}} \int_{\left(\mathcal{O}^{*}\right)^{2}} \int_{\sqrt{c}}^{\infty} \sum_{t_{1}, t_{2} \in \mathbb{Q}^{*}}\left|f\left(\left(\begin{array}{cc}
\left(a^{2} s_{1} s_{2} t_{1}\right)^{-1} & 0 \\
0 & a^{2} s_{1} s_{2} t_{2}
\end{array}\right) x w y\right)\right| \frac{d^{*} a}{a^{2}} d s_{1} d s_{2} d x d y \\
& =\frac{1}{2} \int_{U(\mathbb{A})^{2}} \int_{\left(\mathcal{O}^{*}\right)^{2}} \int_{c}^{\infty} \sum_{t_{1}, t_{2} \in \mathbb{Q}^{*}}\left|f\left(\left(\begin{array}{cc}
\left(a s_{1} s_{2} t_{1}\right)^{-1} & 0 \\
0 & a s_{1} s_{2} t_{2}
\end{array}\right) x w y\right)\right| \frac{d^{*} a}{a} d s_{1} d s_{2} d x d y .
\end{aligned}
$$

We will show that this is bounded by a constant multiple of $\|f\|_{1}$. As pointed out before we may assume that $f$ is of the form (27). In particular we may assume that $K=\boldsymbol{K}_{\text {fin. }}$. We can then remove the $s_{i}$ 's from the integration to get

$$
\begin{aligned}
\int_{U(\mathbb{A})^{2}} & \int_{c}^{\infty} \sum_{t_{1}, t_{2} \in \mathbb{Q}^{*}}\left|f\left(\left(\begin{array}{cc}
\left(a t_{1}\right)^{-1} & 0 \\
0 & a t_{2}
\end{array}\right) x w y\right)\right| \frac{d^{*} a}{a} d x d y \\
= & \int_{c}^{\infty} \sum_{t_{1}, t_{2} \in \mathbb{Q}^{*}} \int_{U(\mathbb{R})^{2}}\left|f_{\infty}\left(\left(\begin{array}{cc}
\left(r \sqrt{N} a t_{1}\right)^{-1} & 0 \\
0 & (r \sqrt{N})^{-1} a t_{2}
\end{array}\right) x_{\infty} w y_{\infty}\right)\right| d x_{\infty} d y_{\infty} \\
& \int_{U\left(\mathbb{A}_{\mathrm{fin}}\right)^{2}} T_{r, N}\left(\left(\begin{array}{cc}
t_{1}^{-1} & 0 \\
0 & t_{2}
\end{array}\right) x_{\mathrm{fin}} w y_{\mathrm{fin}}\right) d x_{\mathrm{fin}} d y_{\mathrm{fin}} \frac{d^{*} a}{a} .
\end{aligned}
$$

Only $t_{1}^{-1} t_{2}= \pm r^{2} N$ contribute, so letting $t=r N t_{1}$ we can rewrite this as (the sum 
over both possible sign choices of)

$$
\begin{aligned}
& \int_{c}^{\infty} \sum_{t \in \mathbb{Q}^{*}} \int_{U(\mathbb{R})^{2}}\left|f_{\infty}\left(\left(\begin{array}{cc}
\sqrt{N}(a t)^{-1} & 0 \\
0 & \pm \sqrt{N}^{-1}{ }_{a t}
\end{array}\right) x_{\infty} w y_{\infty}\right)\right| d x_{\infty} d y_{\infty} \\
& \int_{U\left(\mathbb{A}_{\mathrm{fin}}\right)^{2}} T_{r, N}\left(\left(\begin{array}{cc}
r N t^{-1} & 0 \\
0 & r t
\end{array}\right) x_{\mathrm{fin}} w y_{\mathrm{fin}}\right) d x_{\mathrm{fin}} d y_{\mathrm{fin}} \frac{d^{*} a}{a} \\
& =\sum_{t \in \mathbb{Q}^{*}} \int_{U(\mathbb{R})^{2}} \int_{c|t| / \sqrt{N}}^{\infty} \frac{|t|}{\sqrt{N}}\left|f_{\infty}\left(\operatorname{sgn} t\left(\begin{array}{cc}
a^{-1} & 0 \\
0 & \pm a
\end{array}\right) x_{\infty} w y_{\infty}\right)\right| d x_{\infty} d y_{\infty} \\
& \int_{U\left(\mathbb{A}_{\mathrm{fin}}\right)^{2}} T_{r, N}\left(\left(\begin{array}{cc}
r N t^{-1} & 0 \\
0 & r t
\end{array}\right) x_{\mathrm{fin}} w y_{\mathrm{fin}}\right) d x_{\mathrm{fin}} d y_{\mathrm{fin}} \frac{d^{*} a}{a} \\
& =\int_{0}^{\infty} \sum_{t \in \mathbb{Q}^{*}:|t|<\sqrt{N} a / c} \frac{|t|}{\sqrt{N}} \int_{U(\mathbb{R})^{2}}\left|f_{\infty}\left(\operatorname{sgn} t\left(\begin{array}{cc}
a_{0}^{-1} & 0 \\
0 & \pm a
\end{array}\right) x_{\infty} w y_{\infty}\right)\right| d x_{\infty} d y_{\infty} \\
& \int_{U\left(\mathbb{A}_{\mathrm{fin}}\right)^{2}} T_{r, N}\left(\left(\begin{array}{cc}
r N t^{-1} & 0 \\
0 & r t
\end{array}\right) x_{\mathrm{fin}} w y_{\mathrm{fin}}\right) d x_{\mathrm{fin}} d y_{\mathrm{fin}} \frac{d^{*} a}{a} .
\end{aligned}
$$

By Proposition 3.3 the last integral vanishes unless $t \in \mathbb{Z}$ in which case it is bounded by $\frac{N}{|t|} \sigma_{0}(\operatorname{gcd}(N,|t|))$. We can therefore bound the entire expression by

$$
\int_{0}^{\infty} \sum_{\substack{0 \neq t \in \mathbb{Z}: \\
|t|<\sqrt{N} a / c}} \sqrt{N} \sigma_{0}(\operatorname{gcd}(N, t)) \int_{U(\mathbb{R})^{2}}\left|f_{\infty}\left(\operatorname{sgn} t\left(\begin{array}{cc}
a_{0}^{-1} & 0 \\
0 & \pm a
\end{array}\right) x_{\infty} w y_{\infty}\right)\right| d x_{\infty} d y_{\infty} \frac{d^{*} a}{a}
$$

We have

$$
\sqrt{N} \sum_{1 \leq k \leq \sqrt{N} a / c} \sigma_{0}(\operatorname{gcd}(k, N))=\sqrt{N} \sum_{d \mid N} \sum_{k \leq \sqrt{N} a / c: d \mid k} 1 \leq \frac{a}{c} \sum_{d \mid N} \frac{N}{d}=\frac{a \sigma_{1}(N)}{c} .
$$

Therefore, the expression above is bounded by

$$
\frac{\sigma_{1}(N)}{c} \int_{\mathbb{R}^{*}} \int_{U(\mathbb{R})^{2}}\left|f_{\infty}\left(\left(\begin{array}{cc}
a^{-1} & 0 \\
0 & \pm a
\end{array}\right) x_{\infty} w y_{\infty}\right)\right| d x_{\infty} d y_{\infty} d^{*} a
$$

Observe that

$$
\frac{\sigma_{1}(N)}{\operatorname{deg}\left(T_{r, N}\right)} \leq \frac{N \prod_{p \mid N}\left(1-p^{-1}\right)^{-1}}{N \prod_{p \mid N}\left(1+p^{-1}\right)}=\prod_{p \mid N}\left(1-p^{-2}\right)^{-1} \leq \zeta(2)
$$

Using (18) we can therefore bound (28) by

$$
\frac{\pi \zeta(2)}{c} \operatorname{deg}\left(T_{r, N}\right)\left\|f_{\infty}\right\|_{1}
$$

as required. 
Hyperbolic contribution. Assume that $f$ is of type (27). Then upon changing the variable $t$ by a scalar matrix, the seminorms (7) and (6) are given by

$$
\sum_{t \in T(\mathbb{Q})} \lambda_{t, S} \int_{U(\mathbb{R})}\left|f_{\infty}\left(t_{\infty}^{1} u\right)\right| d u \int_{U\left(\mathbb{A}_{\text {fin }}\right)} T_{r, N}\left(\left(\begin{array}{cc}
r & 0 \\
0 & r
\end{array}\right) t u\right) d u
$$

and

$$
\begin{aligned}
& \sum_{t \in T(\mathbb{Q})} \int_{U(\mathbb{R})}\left|f_{\infty}\left(t_{\infty}^{1} u\right) \omega_{\infty}(t, u)\right| d u \int_{U\left(\mathbb{A}_{\mathrm{fin}}\right)} T_{r, N}\left(\left(\begin{array}{cc}
r & 0 \\
0 & r
\end{array}\right) t u\right) d u \\
& \quad+\sum_{t \in T(\mathbb{Q})} \int_{U(\mathbb{R})}\left|f_{\infty}\left(t_{\infty}^{1} u\right)\right| d u \int_{U\left(\mathbb{A}_{\mathrm{fin}}\right)} T_{r, N}\left(\left(\begin{array}{cc}
r & 0 \\
0 & r
\end{array}\right) t u\right) \sum_{p<\infty}\left|\omega_{p}(t, u)\right| d u,
\end{aligned}
$$

respectively. Combining Proposition 3.3 and Lemma 3.7 the invariant term (29) is bounded by

$$
\mu\left(f_{\infty}\right) \sum_{t \in \mathscr{D}(N)} \lambda_{t, S} \min \left(\left|t_{1}\right|,\left|t_{2}\right|\right)
$$

for some seminorm $\mu$ on $\mathcal{C}\left(G(\mathbb{R})^{1}\right)$ where

$$
\mathscr{D}(N)=\left\{t=\left(\begin{array}{cc}
t_{1} & 0 \\
0 & t_{2}
\end{array}\right) \in T(\mathbb{Q}): \operatorname{det}(t)= \pm N, t_{1}, t_{2} \in \mathbb{Z}\right\} .
$$

Clearly, $\lambda_{t, S} \leq \log N$ if $t \in \mathscr{D}(N) \backslash Z(\mathbb{Q})$, and therefore the sum is bounded by

$$
2 \lambda_{1, S}+2 \sigma_{0}(N) \sqrt{N} \log N .
$$

On the other hand, again by Proposition 3.3 the non-invariant term (30) is bounded by

$$
\sum_{t \in \mathcal{D}(N)}\left|t_{1}\right|\left(\log t_{1}+|S|-1\right) \int_{U(\mathbb{R})}\left|f_{\infty}\left(t_{\infty}^{1} u\right)\right| d u+\sum_{t \in \mathcal{D}(N)}\left|t_{1}\right| \int_{U(\mathbb{R})}\left|f_{\infty}\left(t_{\infty}^{1} u\right) \omega_{\infty}(t, u)\right| d u
$$

By Lemma 3.7 this is bounded by

$2 \sum_{t \in \mathscr{D}(N)} \mu\left(f_{\infty}\right) \min \left(\left|t_{1}\right|,\left|t_{2}\right|\right)(\log N+|S|+1) \leq 4 \mu\left(f_{\infty}\right) \sigma_{0}(N) \sqrt{N}(\log N+|S|+1)$.

It remains to invoke the crude estimate

$$
\sigma_{0}(N) \sqrt{N} \log N=O\left(\operatorname{deg} T_{r, N}\right) .
$$

Remark 2. The proof shows in fact that the seminorms (5), (6) and (7) are continuous with respect to $|\|\cdot\||_{3}$. 


\section{References}

[Art85] J. Arthur, A measure on the unipotent variety. Canad. J. Math. 37 (1985), 1237-1274. Zbl 0589.22016 MR 828844

[Art86] J. Arthur, On a family of distributions obtained from orbits. Canad. J. Math. 38 (1986), 179-214. Zbl 0593.22015 MR 835041

[Art05] J. Arthur, An introduction to the trace formula. In Harmonic analysis, the trace formula, and Shimura varieties, Clay Math. Proc. 4, Amer. Math. Soc., Providence, RI, 2005, 1-263. Zbl 1152.11021 MR 2192011

[DW78] D. L. DeGeorge and N. R. Wallach, Limit formulas for multiplicities in $L^{2}(\Gamma \backslash G)$. Ann. of Math. (2) 107 (1978), 133-150. Zbl 0397.22007 MR 0492077

[DW79] D. L. DeGeorge and N. R. Wallach, Limit formulas for multiplicities in $L^{2}(\Gamma \backslash G)$ II. The tempered spectrum. Ann. of Math. (2) 109 (1979), 477-495. Zbl 0482.43006 MR 534759

[De186] P. Delorme, Formules limites et formules asymptotiques pour les multiplicités dans $L^{2}(G / \Gamma)$. Duke Math. J. 53 (1986), 691-731. Zbl 0623.22012 MR 860667

[DKV79] J. J. Duistermaat, J. A. C. Kolk, and V. S. Varadarajan, Spectra of compact locally symmetric manifolds of negative curvature. Invent. Math. 52 (1979), 27-93; Erratum ibid. 54 (1979), 101. Zbl 0434.58019 MR 532745

[FL] Tobias Finis and Erez Lapid, On the continuity of Arthur's trace formula: the semisimple terms. Compositio Math., to appear.

[FLM] Tobias Finis, Erez Lapid, and Werner Müller, On the spectral side of Arthur's trace formula - absolute convergence. Preprint 2010.

[FLM09] T. Finis, E. M. Lapid, and W. Müller, The spectral side of Arthur's trace formula. Proc. Natl.Acad. Sci. USA 106(2009), 15563-15566.Zbl 1203.11042 MR 2550527

[Ge196] S. Gelbart, Lectures on the Arthur-Selberg trace formula. Univ. Lecture Ser. 9, Amer. Math. Soc., Providence, R.I., 1996. Zbl 0876.11022 MR 1410260

[GJ79] S. Gelbart and H. Jacquet, Forms of GL(2) from the analytic point of view. In Automorphic forms, representations and L-functions, Proc. Sympos. Pure Math. 33, Part 1, Amer. Math. Soc., Providence, R.I., 1979, 213-251. Zbl 0409.22013 MR 546600

[GJ72] R. Godement and H. Jacquet, Zeta functions of simple algebras. Lecture Notes in Mathem. 260, Springer-Verlag, Berlin 1972. Zbl 0244.12011 MR 0342495

[Hof08] W. Hoffmann, Geometric estimates for the trace formula. Ann. Global Anal. Geom. 34 (2008), 233-261. Zbl 1165.11051 MR 2434856

[KL06] A. Knightly and C. Li, Traces of Hecke operators. Math. Surveys Monogr. 133, Amer. Math. Soc., Providence, R.I., 2006. Zbl 1120.11024 MR 2273356

[Lan76] R. P. Langlands, On the functional equations satisfied by Eisenstein series. Lecture Notes in Math. 544, Springer-Verlag, Berlin 1976. Zbl 0332.10018 MR 0579181

[Lan04] R. P. Langlands, Beyond endoscopy. In Contributions to automorphic forms, geometry, and number theory, Johns Hopkins University Press, Baltimore, MD, 2004, 611-697. Zbl 1078.11033 MR 2058622 
[Lan07] R. P. Langlands, Un nouveau point de repère dans la théorie des formes automorphes. Canad. Math. Bull. 50 (2007), 243-267. Zbl 1138.11019 MR 2317447

[Sel56] A. Selberg, Harmonic analysis and discontinuous groups in weakly symmetric Riemannian spaces with applications to Dirichlet series. J. Indian Math. Soc. (N.S.) 20 (1956), 47-87. Zbl 0072.08201 MR 0088511

[Shi71] G. Shimura, Introduction to the arithmetic theory of automorphic functions. Publ. Math. Soc. Japan. 11, Princeton University Press, Princeton, Iwanami Shoten, Tokyo 1971. Zbl 0221.10029 MR 0314766

[Ven04] A. Venkatesh, Beyond endoscopy and special forms on GL(2). J. Reine Angew. Math. 577 (2004), 23-80. Zbl 1061.22019 MR 2108212

Received September 2, 2008; revised April 20, 2009

T. Finis, Mathematisches Institut, Heinrich-Heine-Universität Düsseldorf, Universitätsstraße 1, 40225 Düsseldorf, Germany

E-mail: finis@math.uni-duesseldorf.de

E. Lapid, Institute of Mathematics, Manchester Building (Giv'at-Ram), Hebrew University of Jerusalem, Jerusalem 91904, Israel

E-mail: erezla@math.huji.ac.il 